\title{
Aww: The Emotion of Perceiving Cuteness
}

\author{
Ralf C. Buckley * \\ School of Environment, International Chair in Ecotourism Research, Griffith University, Gold Coast, QLD, Australia
}

Keywords: cute, kawaii, cuteness, evolution, language

Emotions are one of the core components of human biology and behavior, linking senses, thoughts, and actions. In particular, emotions are one of the main components of human communications, including: facial expressions, postures and body language, pheromones and chemosignals, non-linguistic vocalizations, and spoken language (Lambie, 2009; Niedenthal and Brauer, 2012; Dehaene, 2014; Van den Stock and de Gelder, 2014; Lench et al., 2015; Ekman, 2016; Haviland-Jones et al., 2016; Martinez et al., 2016).

Neurophysiologists analyse human emotions as complex interlinked continua of neurological and hormonal signals and processes (Dehaene, 2014; Etkin et al., 2015; Bray, 2016). The social sciences, however, rely on the subtleties of language to differentiate and communicate the fine distinctions between closely related emotions (Johnson-Laird and Oatley, 1989; Ekman, 2016; Lakoff, 2016; Tissari, 2016). Some of these distinctions are very subtle, and we use languages to communicate those subtleties to each other.

For example, the emotion of fear is heavily studied (Buckley, 2016; Méndez-Bértolo et al., 2016), and there is a large family of English words related to the concept. These include: nouns describing specific emotions, such as anxiety, apprehension, trepidation, fright, terror, panic, and

\section{OPEN ACCESS}

Edited by:

Erica Cosentino,

Ruhr University Bochum, Germany

Reviewed by:

Anna Welpinghus,

Technical University of Dortmund,

Germany

*Correspondence:

Ralf C. Buckley

ralf.c.buckley@gmail.com;

r.buckley@griffith.edu.au

Specialty section:

This article was submitted to

Theoretical and Philosophical

Psychology,

a section of the journal

Frontiers in Psychology

Received: 07 September 2016

Accepted: 24 October 2016

Published: 10 November 2016

Citation:

Buckley RC (2016) Aww: The Emotion of Perceiving Cuteness. Front. Psychol. 7:1740.

doi: 10.3389/fpsyg.2016.01740 so on; adjectives describing either the source of fear or the person experiencing it, such as frightful or fearful; and adverbs and derived nouns, such as frighteningly and fearfulness.

As in many aspects of human communications, there are sometimes subtle cultural distinctions in the terminologies of communications. Kundera (1980), for example, argues that there is no exact English equivalent for the Czech word litost, a particular category of sadness. In general, however, there is a substantial degree of correspondence, between most modern languages, in the linguistic taxonomies of emotional constructs.

There is, however, one surprising omission. Across a number of major language groups, there are words for cute and cuteness, but no words for the human emotion experienced on perceiving cuteness. Terms such as kawaii in Japanese, or mignon in French, are sufficiently well-known to be adopted across cultures and languages. Indeed, the concept of kawaii includes an entire lexicon of associated subterms such as mori, loli, kei, and decora, which describe styles, fashions and personalities. There are terms for cute across languages with very different roots; moni in Spanish, sudara in Punjabi, ke ai in Chinese.

None of the languages listed above, however, contain a single term for the corresponding emotional response. Kawaii may translate as loveable, but love is not the emotion of cuteness, in the same way that happiness is not the same as awe (Coghlan et al., 2012; Darbor et al., 2015). Research in this field is forced to use portmanteau terms such as cute-emotion, cute-affect, or kawaii-feeling (Nittono et al., 2012; Laohakangvalvit et al., 2016; Nittono, 2016). In popular English, cute-emotion is represented by vocalizations such as $a w w$ or daww. Aww is listed in UK-English dictionaries, but only as an exclamation, not a noun.

Indeed, there is remarkably little published research on this emotion, relative to other human emotions. There are $>1000$ research publications on emotions such as fear (Buckley, 2016; Méndez-Bértolo et al., 2016), applying social, behavioral, physiological, and neurological as well as psychological perspectives. There are apparently $<10$, at least two orders of magnitude fewer, on cute-emotion (Nittono et al., 2012; Laohakangvalvit et al., 2016; Nittono, 2016). 
Why should this matter? Language and terminology are powerful drivers of human attitudes and behaviors (Eskine, 2013), as demonstrated repeatedly in regard to racism (Yoo et al., 2009; Wei et al., 2015), gender (Formanowicz et al., 2015; Garnham et al., 2016; Hansen et al., 2016; Sczesny et al., 2016); crime (Hansen et al., 2016); and mental and physical health, body image and self-esteem (Yoo et al., 2009; Wei et al., 2015; Meadows and Danílsdóttir, 2016). Names, or the lack of names, affect how people think and act.

This linguistic deficiency is particularly surprising, since cute-emotion has considerable biological significance. Cuteemotion is principally a response to neotenic or baby-animal characteristics, such as big round eyes, small size, and softness. These characteristics are involved in human mate selection (Jones and Hill, 1993; Jones et al., 1995; Perrett et al., 1998; Thayer and Dobson, 2013) and human parental care (Sherman et al., 2009; Nittono et al., 2012; Sprengelmeyer et al., 2013; Aragón et al., 2015; Golle et al., 2015; Hahn et al., 2016; though see also Sherman and Haidt, 2011).

Cute-emotion is a distinct and recognizable emotion, that complies with all the standard definitional criteria, such as characteristic hormonal responses, vocalizations, and facial expressions, recognizable across cultures and linked across senses (Röder et al., 2013). Its existence as an emotion, albeit as yet without a name, is recognized in psychological research (Lambie, 2009; Sherman et al., 2009; Winkielman et al., 2009; Sherman and Haidt, 2011; Nittono et al., 2012).

Cute-emotion is widely recognized and exploited in fashion, design (Huang et al., 2016; Noguchi and Tomoike, 2016), and marketing (Nittono et al., 2012; Nenkov and Scott, 2014; Nittono, 2016). In all these fields, commercial enterprises deliberately use cute characteristics to generate cute-emotion responses by their audiences or clientele. This is a powerful and effective approach. Similarly, cartoon movies and video games create cute characters to appeal to audiences and increase sales (Winkielman et al., 2009), and individuals preferentially watch videos of animals with cute characteristics (Nekaris et al., 2013).

Cute-emotion is thus recognized and used widely and pervasively in modern human societies. Despite this, it does not have a name, other than vernacular expressions such as "aww" in English. There are various possible reasons for this. The most plausible is that only modern civilizations have had the luxury of recognizing and responding to cuteness, through the deliberate design, manufacture, marketing and sale of items perceived as cute and used in social interactions. That is, even though the biological functions of cuteness in mate selection and parental care are longstanding, its social functions in peer esteem may be new.

With limited contrary evidence (Scott et al., 2014), biological responses to neotenic characteristics, as expressed in human

\section{REFERENCES}

Achrati, A. (2014). Neoteny, female hominin and cognitive evolution. Rock Art Res. $31,232$.

Aragón, O. R., Clark, M. S., Dyer, R. L., and Bargh, J. A. (2015). Dimorphous expressions of positive emotion: displays of both care and aggression in mate choice and parental care, seem to be evolutionarily conservative, especially since similar characteristics are shared across many other species. The exaggerated representation of neotenic features, which contribute to cuteness in graphical and artistic representations, has precedents in ancient rock art (Achrati, 2014). It seems, therefore, that the biological functions of cute-emotion are not new.

The linguistics are more difficult to trace. The Japanese word kawaii is apparently $>1000$ years old, and the same probably applies for the Chinese term ke ai. It appears, however, that the precise meaning of the term has changed over time. In its earlier uses, it was applied in the literal sense to mean "loveable." The much more specific associations of the modern usage of kawaii, e.g., in dress, fashion and toys, has only evolved recently. The widespread change in clothing styles from traditional kimono to the plethora of Japanese dress fashions such as mori, loli, and kei has taken place within the last half-century. The dissemination and stylistic cross-fertilization between Japanese kawaii fashions and other cultures is equally recent. Adoption of kawaii styles in toys and design has also expanded largely within the last few decades. During this same period of rapid and recent cultural linguistic evolution, the meaning of the term "cute" in English has also become diversified. The term can be used in a pejorative as well as an appreciative sense, and this may have blocked the evolution of a term for cute-emotion.

The use of cute-emotion in social interactions, however, is now widespread and pervasive in Western as well as Eastern cultures and countries. As just one example not mentioned above, marketing materials for wildlife tourism products focus heavily on images of young animals which generate cuteemotion in potential purchasers (Buckley and Mossaz, 2016). Given the significance of cute-emotion in biology, society, and commerce, I propose that it deserves substantially greater attention in psychological research, building on existing studies cited here, and adopting all the methodological approaches used widely in studying other human emotions. I suggest that one possible reason for the limited attention it has received to date, relative to its social significance, is that it has not had a simple and widely recognized formal name. Attitudes and behavior, for research psychologists as for everyone else, are influenced by language. To overcome this obstacle, I propose that the most common English vernacular term, aww, should be adopted as a formal name for cuteemotion.

\section{AUTHOR CONTRIBUTIONS}

The author confirms being the sole contributor of this work and approved it for publication.

response to cute stimuli. Psychol. Sci. 26, 259-273. doi: 10.1177/095679761456 1044

Bray, N. (2016). Behavioural neuroscience: on the defensive. Nat. Rev. Neurosci. 17, 463. doi: $10.1038 / \mathrm{nrn} .2016 .88$

Buckley, R. C. (2016). Qualitative analysis of emotions: fear and thrill. Front. Psychol. 7:1187. doi: 10.3389/fpsyg.2016.01187 
Buckley, R., and Mossaz, A. C. (2016). Decision making by specialist luxury travel agents. Tourism Manag. 55, 133-138. doi: 10.1016/j.tourman.2016.02.002

Coghlan, A., Buckley, R., and Weaver, D. (2012). A framework for analysing awe in tourism experiences. Ann. Tourism Res. 39, 1710-1714. doi: 10.1016/j.annals.2012.03.007

Darbor, K. E., Lench, H. C., Davis, W. E., and Hicks, J. A. (2015). Experiencing versus contemplating: language use during descriptions of awe and wonder. Cogn. Emot. 30, 1188-1196. doi: 10.1080/02699931.2015.1042836

Dehaene, S. (2014). Consciousness and the Brain. New York, NY: Viking.

Ekman, P. (2016). What scientists who study emotion agree about. Perspect. Psychol. Sci. 11, 31-34. doi: 10.1177/1745691615596992

Eskine, K. J. (2013). On the representation and processing of social information in grounded cognitive systems: why terminology matters. Front. Psychol. 4:180. doi: $10.3389 /$ fpsyg.2013.00180

Etkin, A., Büchel, C., and Gross, J. J. (2015). The neural bases of emotion regulation. Nat. Rev. Neurosci. 16, 693-700. doi: 10.1038/nrn4044

Formanowicz, M. M., Cisłak, A., Horvath, L. K., and Sczesny, S. (2015). Capturing socially motivated linguistic change: how the use of gender-fair language affects support for social initiatives in Austria and Poland. Front. Psychol. 6:1617. doi: 10.3389/fpsyg.2015.01617

Garnham, A., Oakhill, J., Von Stockhausen, L., and Sczesny, S. (2016). Language, cognition, and gender. Front. Psychol. 7:772. doi: 10.3389/fpsyg.2016.00772

Golle, J., Probst, F., Mast, F. W., and Lobmaier, J. S. (2015). Preference for cute infants does not depend on their ethnicity or species: evidence from hypothetical adoption and donation paradigms. PLOS ONE 10:e0121554. doi: 10.1371/journal.pone.0121554

Hahn, A. C., Symons, L. A., Kredel, T., Hanson, K., Hodgson, L., Schiavone, L., et al. (2016). Early and late event-related potentials are modulated by infant and adult faces of high and low attractiveness. Soc. Neurosci. 11, 207-220. doi: 10.1080/17470919.2015.1059361

Hansen, K., Littwitz, C., and Sczesny, S. (2016). The social perception of heroes and murderers: effects of gender-inclusive language in media reports. Front. Psychol. 7:369. doi: 10.3389/fpsyg.2016.00369

Haviland-Jones, J. M., McGuire, T. R., and Wilson, P. (2016). Testing for individual differences in the identification of chemosignals for fear and happy: phenotypic super-detectors, detectors and non-detectors. PLOS ONE 11:e0154495. doi: 10.1371/journal.pone.0154495

Huang, S. M., Li, W. J., and Tung, S. C. (2016). "A study of attributes of affective quality affecting judgment of beauty for simple graphic user interfaces," in International Conference of Design, User Experience, and Usability (Cham: Springer International Publishing), 16-24.

Johnson-Laird, P. N., and Oatley, K. (1989). The language of emotions: an analysis of a semantic field. Cogn. Emot. 3, 81-123. doi: 10.1080/02699938908408075

Jones, D., Brace, C. L., Jankowiak, W., Laland, K. N., Musselman, L. E., Langlois, J. H., et al. (1995). Sexual selection, physical attractiveness, and facial neoteny: cross-cultural evidence and implications. Curr. Anthropol. 36, 723-748. doi: $10.1086 / 204427$

Jones, D., and Hill, K. (1993). Criteria of facial attractiveness in five populations. Hum. Nat. 4, 271-296. doi: 10.1007/BF02692202

Kundera, M. (1980). The Unbearable Lightness of Being. New York, NY: Knopf.

Lakoff, G. (2016). Language and emotion. Emot. Rev. 8, 269-273. doi: 10.1177/ 1754073915595097

Lambie, J. A. (2009). Emotion experience, rational action, and self-knowledge. Emot. Rev. 1, 272-280. doi: 10.1177/1754073909103596

Laohakangvalvit, T., Iida, I., Charoenpit, S., and Ohkura, M. (2016). "The study of kawaii feeling by using eye tracking," in Advances in Affective and Pleasurable Design (Cham: Springer International Publishing), 185-197.

Lench, H. C., Bench, S. W., Darbor, K. E., and Moore, M. (2015). A functionalist manifesto: goal-related emotions from an evolutionary perspective. Emot. Rev. 7, 90-98. doi: 10.1177/1754073914553001

Martinez, L., Falvello, V. B., Aviezer, H., and Todorov, A. (2016). Contributions of facial expressions and body language to the rapid perception of dynamic emotions. Cogn. Emot. 30, 939-952. doi: 10.1080/02699931.2015.1035229

Meadows, A., and Daníelsdóttir, S. (2016). What's in a word? On weight stigma and terminology. Front. Psychol. 7:1527. doi: 10.3389/fpsyg.2016.01527

Méndez-Bértolo, C., Moratti, S., Toledano, R., Lopez-Sosa, F., Martínez-Alvarez, R., Mah, Y. H., et al. (2016). A fast pathway for fear in human amygdala. Nat. Neurosci. 19, 1041-1049. doi: 10.1038/nn.4324

Nekaris, K. A.-I., Campbell, N., Coggins, T. G., Rode, E. J., and Nijman, V. (2013). Tickled to death: analysing public perceptions of 'cute' videos of threatened species (slow lorises, Nycticebus spp.) on web 2.0 Sites. PLoS ONE 8:e69215. doi: 10.1371/journal.pone.0069215

Nenkov, G. Y., and Scott, M. L. (2014). "So cute I could eat it up": priming effects of cute products on indulgent consumption. J. Consum. Res. 41, 326-341. doi: $10.1086 / 676581$

Niedenthal, P. M., and Brauer, M. (2012). Social functionality of human emotion. Annu. Rev. Psychol. 63, 259-285. doi: 10.1146/annurev.psych.121208.131605

Nittono, H. (2016). The two-layer model of 'kawaii': a behavioural science framework for understanding kawaii and cuteness. East Asian J. Pop. Cult. 2, 79-95. doi: 10.1386/eapc.2.1.79_1

Nittono, H., Fukushima, M., Yano, A., and Moriya, H. (2012). The power of kawaii: viewing cute images promotes a careful behavior and narrows attentional focus. PLoS ONE 7:e46362 doi: 10.1371/journal.pone.0046362

Noguchi, Y., and Tomoike, K. (2016). Strongly-motivated positive affects induce faster responses to local than global information of visual stimuli: an approach using large-size Navon letters. Sci. Rep. 6:19136. doi: 10.1038/srep19136

Perrett, D. I., Lee, K. J., Penton-Voak, I., Rowland, D., Yoshikawa, S., Burt, D. M., et al. (1998). Effects of sexual dimorphism on facial attractiveness. Nature 394, 884-887.

Röder, S., Fink, B., Feinberg, D. R., and Neave, N. (2013). Facial visualizations of women's voices suggest a cross-modality preference for femininity. Evol. Psychol. 11:147470491301100119. doi: 10.1177/147470491301100119

Scott, I. M., Clark, A. P., Josephson, S. C., Boyette, A. H., Cuthill, I. C., Fried, R. L., et al. (2014). Human preferences for sexually dimorphic faces may be evolutionarily novel. Proc. Natl. Acad. Sci.U.S.A. 111, 14388-14393. doi: 10.1073/pnas.1409643111

Sczesny, S., Formanowicz, M., and Moser, F. (2016). Can gender-fair language reduce gender stereotyping and discrimination? Front. Psychol. 7:25. doi: 10.3389/fpsyg.2016.00025

Sherman, G. D., and Haidt, J. (2011). Cuteness and disgust: the humanizing and dehumanizing effects of emotion. Emot. Rev. 3, 245-251. doi: $10.1177 / 1754073911402396$

Sherman, G. D., Haidt, J., and Coan, J. A. (2009). Viewing cute images increases behavioral carefulness. Emotion 9, 282. doi: 10.1037/a0014904

Sprengelmeyer, R., Lewis, J., Hahn, A., and Perrett, D. I. (2013). Aesthetic and incentive salience of cute infant faces: studies of observer sex, oral contraception and menstrual cycle. PLoS ONE 8:e65844. doi: 10.1371/journal.pone.0065844

Thayer, Z. M., and Dobson, S. D. (2013). Geographic variation in chin shape challenges the universal facial attractiveness hypothesis. PLOS ONE 8:e60681. doi: 10.1371/journal.pone.0060681

Tissari, H. (2016). Current emotion research in English linguistics: words for emotions in the history of English. Emot. Rev. doi: 10.1177/1754073916632064. [Epub ahead of print].

Van den Stock, J., and de Gelder, B. (2014). Face identity matching is influenced by emotions conveyed by face and body. Front. Hum. Neurosci. 8:53. doi: 10.3389/fnhum.2014.00053

Wei, M., Liang, Y. S., Du, Y., Botello, R., and Li, C. I. (2015). Moderating effects of perceived language discrimination on mental health outcomes among Chinese international students. Asian Am. J. Psychol. 6, 213-222. doi: 10.1037/aap0000021

Winkielman, P., McIntosh, D. N., and Oberman, L. (2009). Embodied and disembodied emotion processing: learning from and about typical and autistic individuals. Emot. Rev. 1, 178-190. doi: 10.1177/17540739081 00442

Yoo, H. C., Gee, G. C., and Takeuchi, D. (2009). Discrimination and health among Asian American immigrants: disentangling racial from language discrimination. Soc. Sci. Med. 68, 726-732. doi: 10.1016/j.socscimed.2008. 11.013

Conflict of Interest Statement: The author declares that the research was conducted in the absence of any commercial or financial relationships that could be construed as a potential conflict of interest.

Copyright (C) 2016 Buckley. This is an open-access article distributed under the terms of the Creative Commons Attribution License (CC BY). The use, distribution or reproduction in other forums is permitted, provided the original author(s) or licensor are credited and that the original publication in this journal is cited, in accordance with accepted academic practice. No use, distribution or reproduction is permitted which does not comply with these terms. 